\title{
An Experimental Approach to Determine the Effect of Different Orientation of Dimples on Flat Plates
}

\author{
Amjad Khan ${ }^{1}$, Mohammed Zakir Bellary ${ }^{1}$, Mohammad Ziaullah ${ }^{1}$, Abdul Razak Kaladgi ${ }^{2}$, \\ ${ }^{1}$ Department of Electronics and communication Engineering, P.A College of Engineering, Mangalore, Karnataka, India \\ ${ }^{2}$ Department of Mechanical Engineering, P.A College of Engineering, Mangalore, Karnataka, India
}

Email address

amjad_ece@pace.edu.in (A. Khan),mdzakir87@gmail.com (M. Z. Bellary), mdziya1990@gmail.com (M. Ziaullah), abdulkaladgi@gmail.com (A. R. Kaladgi)

\section{To cite this article:}

Amjad Khan, Mohammed Zakir Bellary, Mohammad Ziaullah, Abdul Razak Kaladgi. An Experimental Approach to Determine the Effect of Different Orientation of Dimples on Flat Plates. American Journal of Electrical Power and Energy Systems. Vol. 4, No. 3, 2015 , pp. $29-33$. doi: $10.11648 /$ j.epes.20150403.12

\begin{abstract}
Dimples play a very important role in the heat transfer enhancement of electronic cooling systems. In the current paper, the flow and heat transfer characteristics of spherical dimples of non uniform diameter were investigated. The experiment was carried out under laminar forced convection conditions using air as a working fluid. The overall Nusselt number and heat transfer coefficient at different dimple structures were obtained for various inlet air flow rates. From the obtained results, it was observed that the heat transfer coefficient and Nusselt number were high for the plate in which the diameter of dimples increases centrally in the direction of flow as compared to the other cases.
\end{abstract}

Keywords: Forced Convection, Electronic Cooling, Dimples, Passive Techniques

\section{Introduction}

The development of integrated electronic devices with increased level of miniaturization, higher performance and output has increased the cooling requirement of chips considerably. As the chip temperature increases, the stability and efficiency Issues will increase and the problem of heat dissipation will become a bottleneck for the development of chips in the electronic industry [1].Passive heat transfer enhancement techniques are used in electronic cooling devices. In these techniques, passive augmented heat transfer devices such as rib-tabulators, concavities (dimples), extended surfaces or fins, and protrusions are used. Among these, the dimples (concavities) can be considered important because they not only enhance/augment heat transfer rate but also produce minimum pressure drop penalties which is important for pumping power requirements [1]. The dimple usually produces vortex pairs, causes flow separation, creates reattachment zones and hence enhances the heat transfer rate. And as they do not protrude into the flow so they contribute less to the foam drag, to produce minimum pressure drop penalties [2]. Another advantage is that in dimple manufacture the removal of material takes place and reduces the cost and weight of the equipments.
Kuethe [3] can be considered as the first person to use dimples on flat surfaces. He observed that the dimples produces rapid or turbulent mixing in the flow, acting as vortex generator \& increases the heat transfer rate. Afanasyev et al [4] conducted an experimental where he used spherical dimples on flat plates. He observed an increase of $30-40 \%$ in the heat transfer rate along with minimum pressure drop. In another study, Chyu et al [5] used tear drop type dimples along with hemispherical dimples to study the heat transfer distribution in the channel and observed a considerable increment in the heat transfer rate for the surfaces having dimples. Mahmood et al [6] conducted an experiment to investigate the effect of dimples on heat transfer using the flow visualization techniques and concluded that the periodic nature of shedding off of vortices is the main cause of enhancement of heat transfer (much more pronounced at the downstream rims of the dimples).Mahmood et al [7] studied the effect of Reynolds number, aspect ratio, temperature ratio $\&$ flow structure in a channel having dimples at the bottom. And observed through the flow visualization techniques that the secondary vortices that are shed off from the dimples become stronger as the non-dimensional channel height to 
dimple diameter (H/D) ratio decreases and increases the local Nusselt number in these regions. Xie et al [8] carried out a numerical investigation to study the effect of heat transfer enhancing devices (pin-fins protrusions \& dimples) on turbine blade tip wall. And concluded that though the dimples have a simple geometry but they are best suited for cooling of blade tip especially at low Reynolds numbers

From the literature, it is very much clear that dimples (vortex generators) have high potential to enhance the heat transfer along with the production of lower pressure drop penalties. The other advantages include low weight, cost and fouling rates [9]; however, most of the researchers conducted numerical or experimental work on spherical dimples of uniform diameter $[5,10]$. Also most of the research is confined to flow in the channel or Internal flow, with a very few studies on external flow [10].So the main aim of this project is to experimentally study the effect of spherical dimples (non-uniform diameter) on aluminum plates under external laminar forced flow conditions .

\section{Experimental Setup}

The fabricated experimental setup used in this study is as shown in the figure below

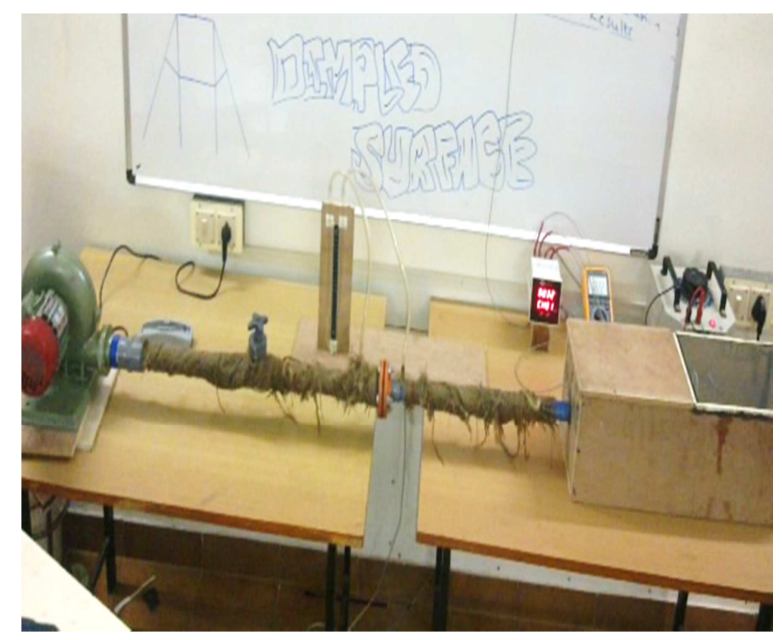

Figure 1. Experimental setup.

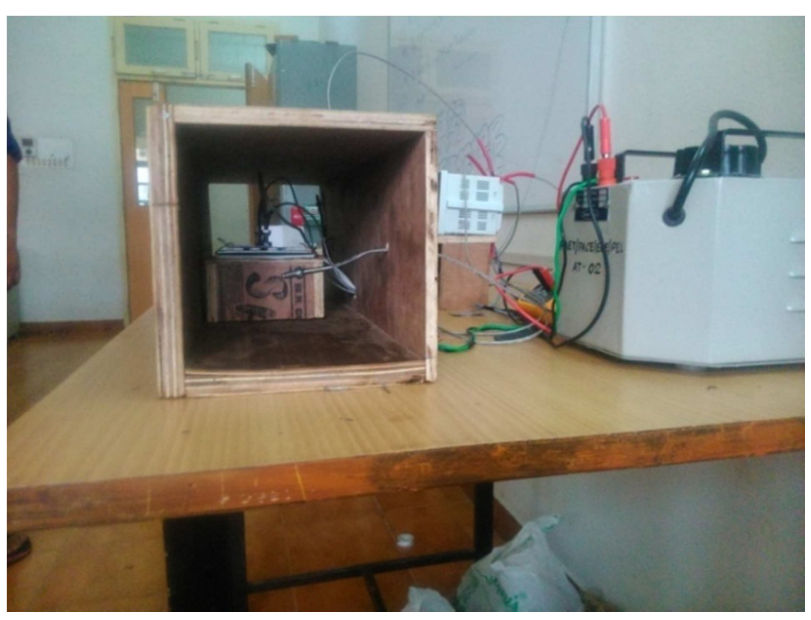

Figure 2. Experimental setup(side view).
The main components of the experimental setup used are aluminum test plates of dimensions $100 \times 100 \times 2 \mathrm{~mm}$, a calibrated orifice flow meter, Strip plate heater (100 watts capacity), Dimmer stat, Digital temperature indicator, voltmeter, and ammeter with $\mathrm{J}$ type thermocouple and a centrifugal blower.

Table 1. Components and Specifications.

\begin{tabular}{ll}
\hline Components & Specification \\
\hline Test plate & $10 \times 10 \times 2 \mathrm{~cm}$ aluminium plates \\
Blower & $110 \mathrm{~W}, 0.4 \mathrm{BHP}, 280 \mathrm{rpm}$ \\
Heater & $100 \mathrm{~W}, 4$ "x4" \\
Dimmer stat & $6 \mathrm{~A}, 230 \mathrm{~V}$ \\
Digital Temperature Indicator & $6 \mathrm{channel}, 12000 \mathrm{C}, 230 \mathrm{~V}$ \\
Orifice plate & $12 \mathrm{~mm}$ dia. \\
Manometer & "U-tube" glass manometer \\
Casing & A wooden casing of size of 8"x8" and \\
& 2feet long. \\
Thermocouple & K-Type, 3000C, 1m long. \\
Digital Multi-meter & Voltmeter, Ammeter \\
\hline
\end{tabular}

\section{Results and Discussion}

Experiments were conducted on aluminum test plates with spherical dimples of non uniform diameters made on flat plates. The dimples were arranged in a staggered fashion with different arrangements like

Case a. Centrally increasing the diameter of dimples in the direction of flow \& maintaining the left \& right column with constant diameter dimples.

Case b.Gradual Increase in the diameter of dimples in the direction flow.

Case c. Gradual Decrease in the diameter of dimples in the direction flow (reverse case).

The data obtained were used to find important heat transfer parameters like Nusselt number, heat transfer coefficient, and heat transfer rate. And the experimental findings have been plotted in the form of graphs, mainly

- Nusselt number(Nu) vs Reynolds number(Re)

- Heat transfer coefficient(h) vs Reynolds number(Re)

- Heat transfer rate Q vs Reynolds number(Re)

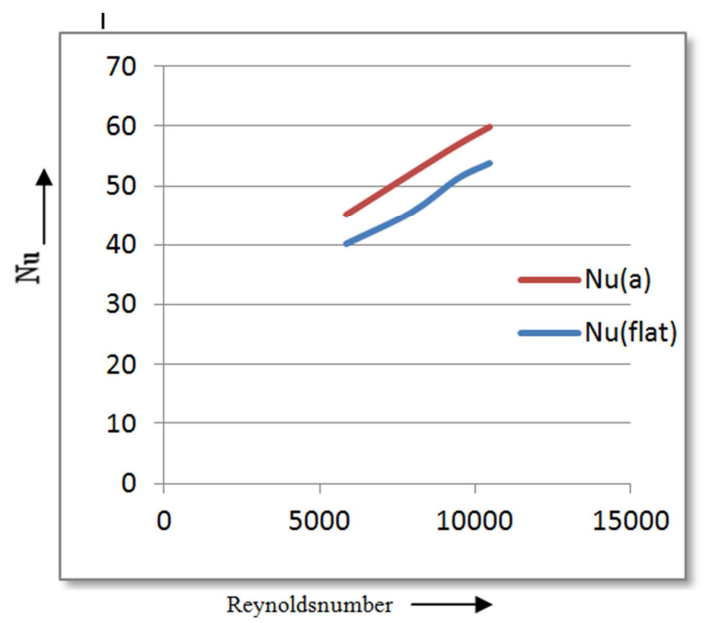

Figure 3. Variation of Nusselt number with Reynolds number(case.a). 


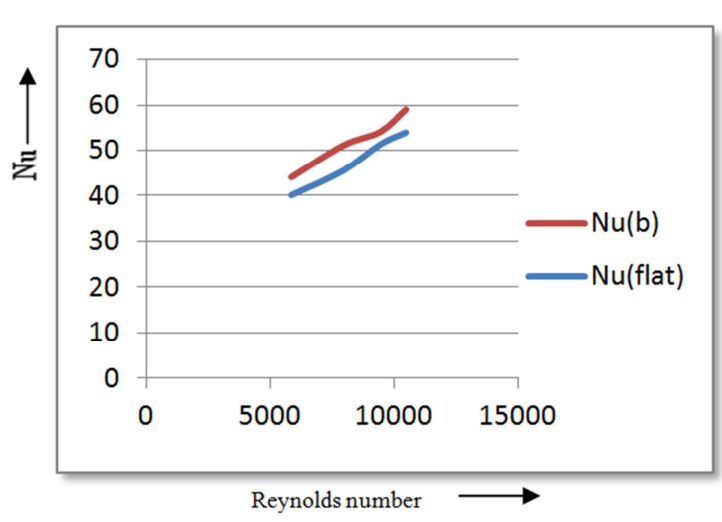

Figure 4. Variation of Nusselt number with Reynolds number(case.b.).

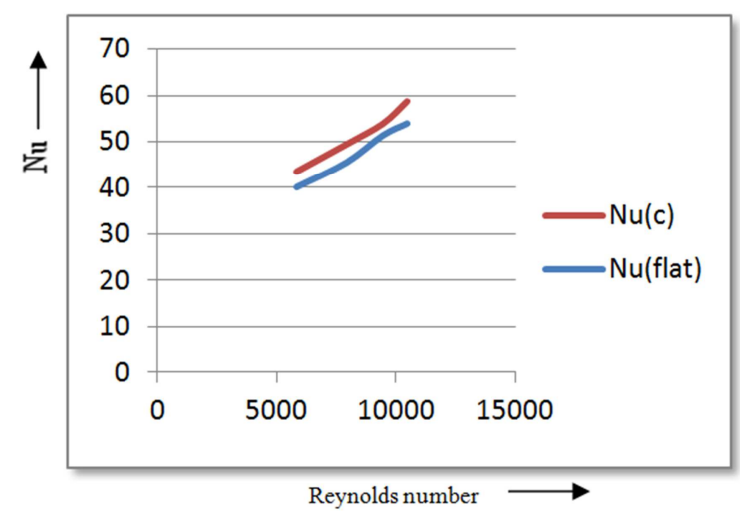

Figure 5. Variation of Nusselt number with Reynolds number(case.c.).

Figure 3, 4, 5 shows variation of Nusselt number 'Nu' with Reynolds number 'Re' for the cases considered. It can be seen that as expected the Nusselt number increases as Reynolds number increases. This is due to direct flow impingement on the downstream boundary of the plate and strengthened flow mixing by vortices at the downstream of the plate $[1,11]$. The formation of vortex pairs, periodically shedding off from the dimples, a large up wash regions with some fluids coming out from the central regions of the dimples are the other reasons of enhancement of Nusselt number \& is more pronounced near the downstream rims of the dimples [6].It can also be seen that the variation in the Nusselt number is gradual with Reynolds number as expected $[12,13,16]$.

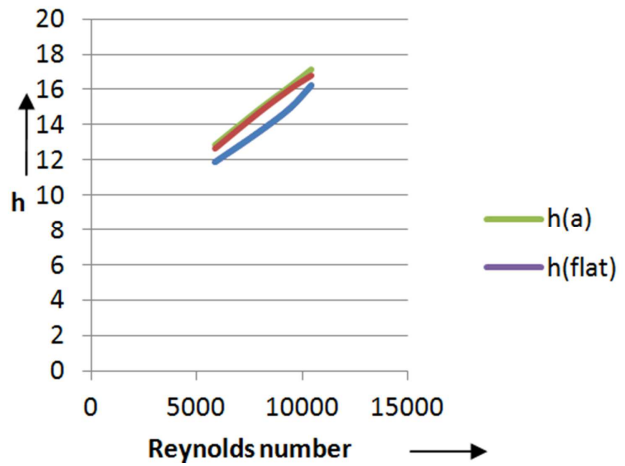

Figure 6. Variation of Heat transfer coefficient with Reynolds number(case.a.).

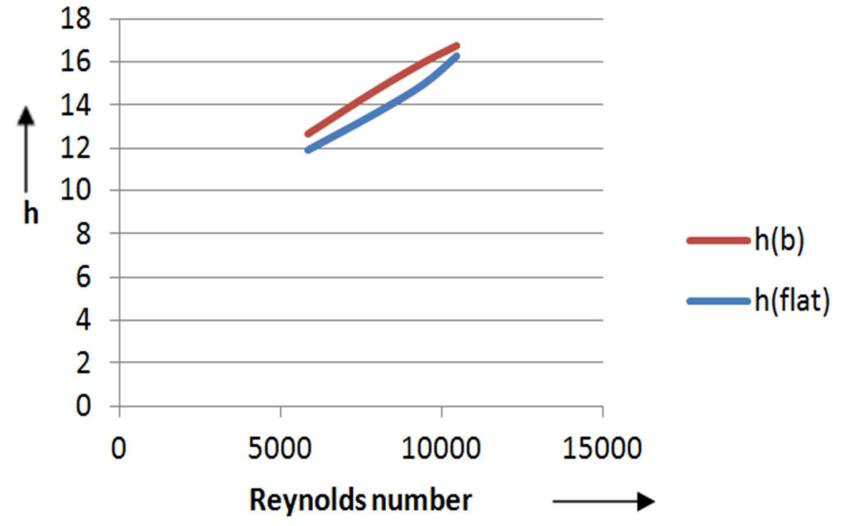

Figure 7. Variation of Heat transfer coefficient with Reynolds number(case.b.).

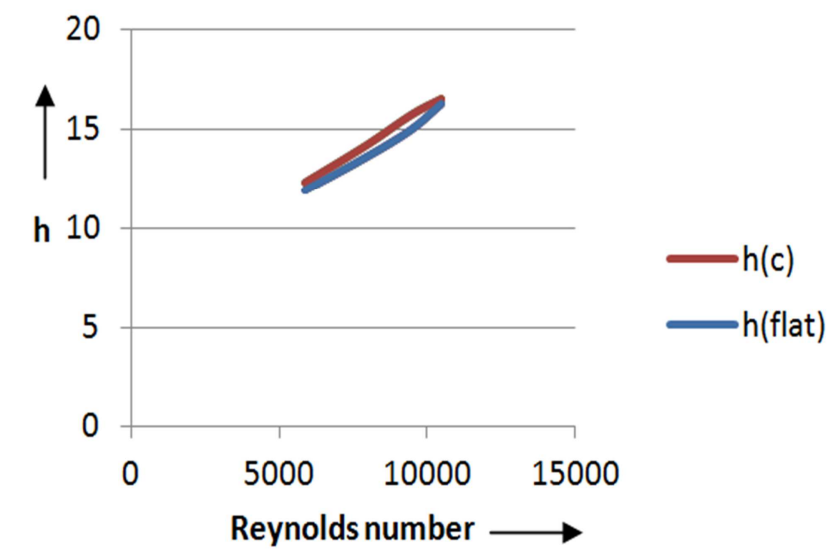

Figure 8. Variation of Heat transfer coefficient with Reynolds number(case.c.).

Figure 6, 7, 8 shows the variation of heat transfer coefficient ' $h$ ' with Reynolds number 'Re' for the various cases considered. It is obvious that ' $h$ ' increases with 'Re'as expected because the development of the thermal boundary layer is delayed or disrupted $\&$ hence enhances the local heat transfer in the reattachment region and wake region and increases the heat transfer coefficient [1].

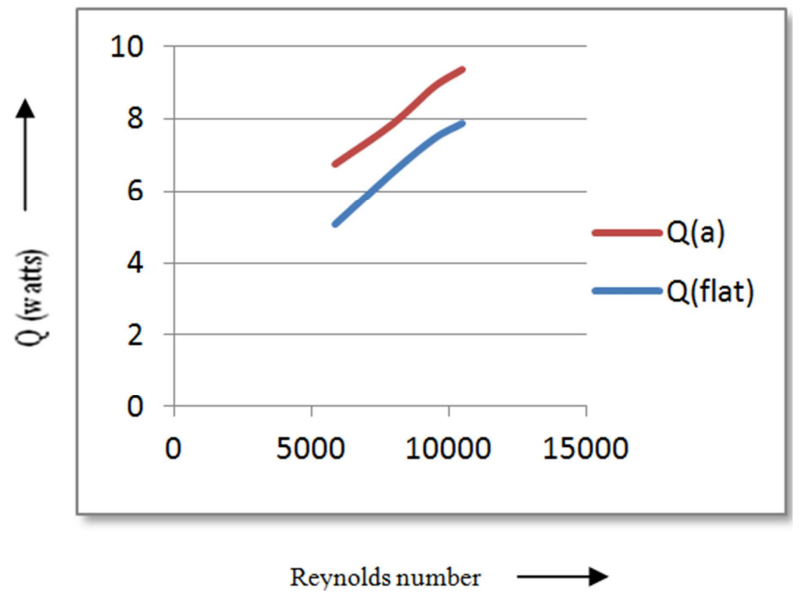

Figure 9. Variation of Heat transfer rate with Reynolds number(case.a.). 


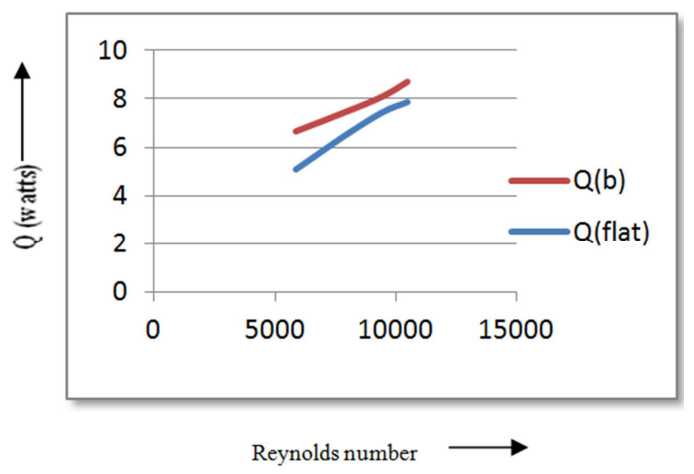

Figure 10. Variation of Heat transfer rate with Reynolds number(case.b.).

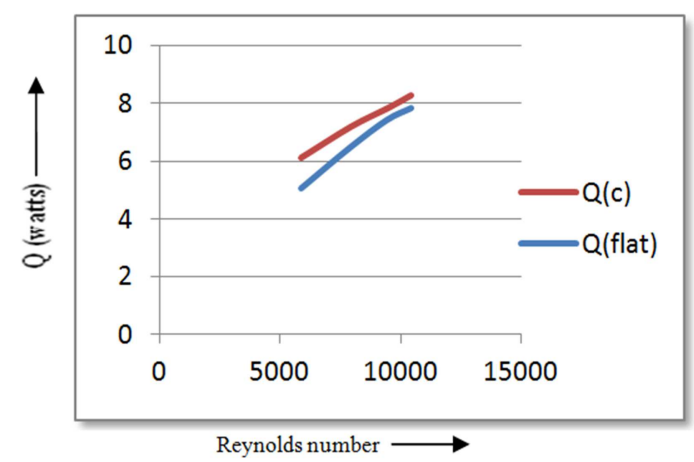

Figure 11. Variation of Heat transfer rate with Reynolds number(case.c.).

Figure 9, 10, 11 shows variation of Heat transfer rate 'Q' with Reynolds number 'Re' for the various cases considered. It can be seen that again ' $Q$ ' increases as 'Re' increases in all the three cases. Because the near-wall turbulent mixing intensity downstream the dimple increases due to the vortex flow shedding from the dimple $[6,15]$ and hence increases the heat transfer rate. It is also seen that ' $Q$ ' is very much higher for case 'a' (dimples diameter decreasing centrally) because of increase in the level of turbulence downstream the dimples. So it can be concluded that case ' $a$ ' helps in better enhancing the heat transfer compared to other cases.

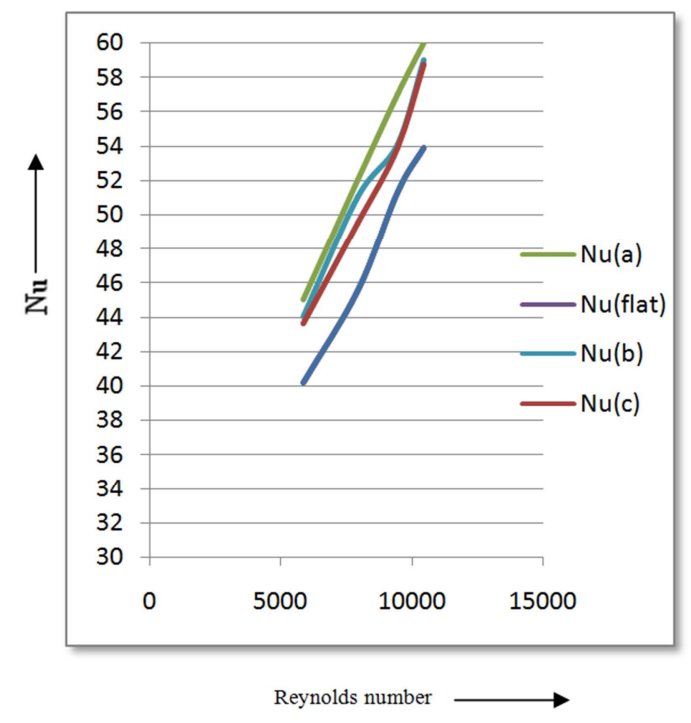

Figure 12. Variation of Nusselt number with Reynolds number.
Figure 12 shows the comparison of Nusselt number ' $\mathrm{Nu}$ ' with Reynolds number ' $\mathrm{Re}$ ' for the all the three cases considered. It can be seen that ' $\mathrm{Nu}$ ' increases as ' $\mathrm{Re}$ ' increases in all the three cases. It can also be seen that the variation of Nusselt number ' $\mathrm{Nu}$ ' for the last two cases is very less especially for high Reynolds number flows. Also the ' $\mathrm{Nu}$ ' is low for the last two cases as compared to the first case may be due the fact that the dimple diameter is not increased or decreased centrally where the pronounce effect of heat transfer will occur. Also this configuration produces strongest turbulent mixing near the wall and hence lowest end wall temperatures [14].

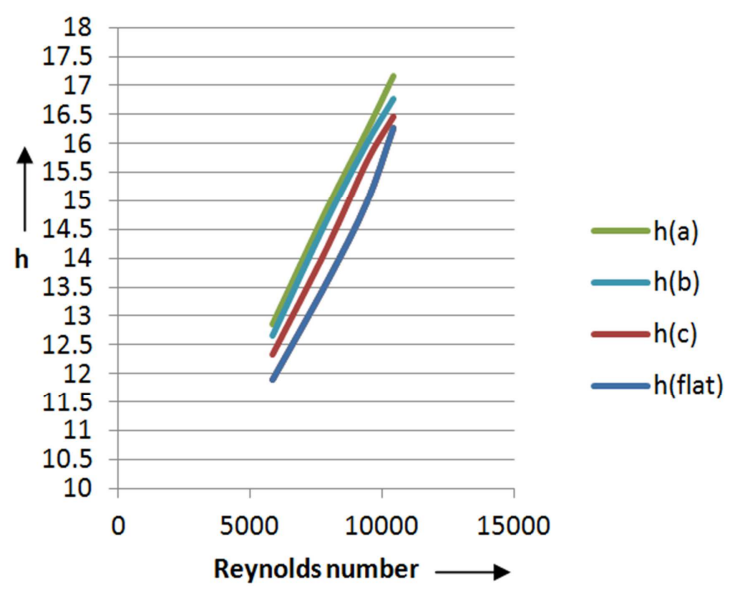

Figure 13. Variation of Heat transfer coefficient with Reynolds number.

Figure 13 shows the comparison of the variation of heat transfer coefficient ' $h$ ' with Reynolds number 'Re' for the various cases considered. It is obvious that ' $h$ ' increases with ' $\mathrm{Re}$ ' as expected and it is also observed that heat transfer coefficient is high for the case' $a$ ' (case of dimple diameter increasing centrally) due to higher turbulent mixing occurring at the central region where the fluid flow rate is highest.

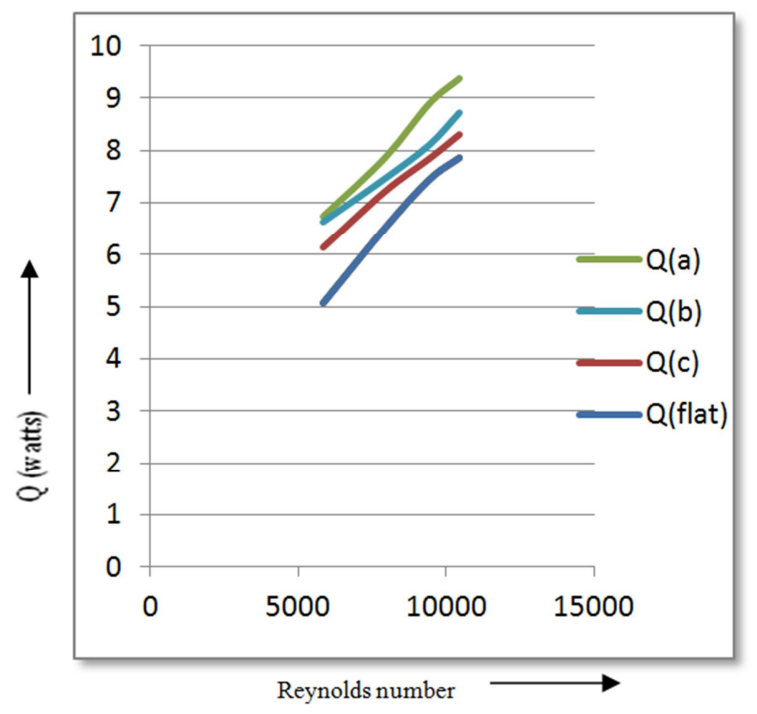

Figure 14. Variation of Heat transfer rate with Reynolds number. 
Figure 14 shows the variation of Heat transfer rate ' $Q$ ' with Reynolds number ' $R e$ ' for the various cases considered. It can be seen that again ' $Q$ ' increases as ' $R e$ ' increases in all the three cases as expected because the vortex flow shedding from the dimples significantly increases the turbulence level in the flow near the downstream wall, and hence increases the convective heat transfer, especially near the rear rim of the dimple. And therefore the end wall temperature of dimple surfaces decreases [14].

\section{Conclusion}

In this experimental work an investigation of the effect of air flow over a flat plate with different diameter dimples is carried out. The main conclusions of the work were:

- Nusselt number increases with Reynolds number for all the three cases of dimple arrangement considered due to direct flow impingement on the downstream boundary and strengthened flow mixing by the vortices at the downstream.

- Case 'a' dimple arrangement has highest Nusselt number because it produces strongest turbulent mixing near the wall and hence produces lowest end wall temperatures. Case' $b$ ' $\&$ 'c' dimple arrangement gives nearly the same value of Nusselt number at high Reynolds numbers.

- Heat transfer coefficient increases with Reynolds number for all the three cases of dimples arrangement considered due to the disruption of the thermal boundary layer development $\&$ hence enhance the local heat transfer in the reattachment and wake regions.

- Case 'a' dimple arrangement gives slightly higher value of heat transfer coefficient as compared to case 'b' \& 'c' dimples.

- Case 'a' dimple arrangement has better heat transfer enhancing capacity as compared to other cases because the level of turbulent mixing is highest in case ' $a$ '. However, the Augmentation depends on the configuration [10].

\section{References}

[1] Zhang, D., Zheng, L., Xie, G., and Xie, Y.,An Experimental Study on Heat Transfer enhancement of Non-Newtonian Fluid in a Rectangular Channel with Dimples/Protrusions, Transactions of the ASME, Vol. 136, pp.021005-10, 2014.

[2] Beves, C.C., Barber, T.J., and Leonardi, E., An Investigation of Flow over Two-Dimensional Circular Cavity. In 15th Australasian Fluid Mechanics Conference, the University of Sydney, Australia, pp.13-17, 2004.

[3] Kuethe A. M., Boundary Layer Control of Flow Separation and Heat Exchange. US Patent No. 1191, 1970.
[4] Afanasyev, V. N., Chudnovsky, Y. P., Leontiev, A. I., and Roganov, P. S., Turbulent flow friction and heat transfer characteristics for spherical cavities on a flat plate. Experimental Thermal Fluid Science, Vol. 7, Issue 1, pp. 1-8, 1993.

[5] Chyu, M.K., Yu, Y., Ding, H., Downs, J.P., and Soechting, F.O., Concavity enhanced heat transfer in an internal cooling passage. In Orlando international Gas Turbine \& Aero engine Congress \& Exhibition, Proceedings of the 1997(ASME paper 97-GT-437), 1997.

[6] Mahmood, G. I., Hill, M. L., Nelson, D. L., Ligrani, P. M., Moon, H. K and Glezer, B., Local heat transfer and flow structure on and above a dimpled surface in a channel. J Turbo mach, Vol.123, Issue 1, pp: 115-23, 2001.

[7] Mahmood, G. I., and Ligrani, P. M., Heat Transfer in a Dimpled Channel: Combined Influences of Aspect Ratio, Temperature Ratio, Reynolds Number, and Flow Structure. Int. J. Heat Mass Transfer, Vol.45, pp.2011-2020, 2002.

[8] Xie, G. N., Sunden, B., and Zhang, W. H., Comparisons of Pins/Dimples Protrusions Cooling Concepts for an Internal Blade Tip-Wall at High Reynolds Numbers. ASME J. Heat Transfer, Vol.133, Issue 6, pp. 0619021-0619029, 2011.

[9] Gadhave, G., and Kumar.P. Enhancement of forced Convection Heat Transfer over Dimple Surface-Review. International Multidisciplinary e - Journal .Vol-1, Issue-2, pp.51-57, 2012

[10] Katkhaw, N., Vorayos, N., Kiatsiriroat, T., Khunatorn, Y., Bunturat, D., and Nuntaphan., A. Heat transfer behavior of flat plate having 450 ellipsoidal dimpled surfaces. Case Studies in Thermal Engineering, vol.2, pp. 67-74, 2014

[11] Patel, I.H., and Borse, S.H. Experimental investigation of heat transfer enhancement over the dimpled surface. International Journal of Engineering Science and Technology, Vol.4, Issue 6, pp.3666-3672, 2012.

[12] Faheem Akhtar, Abdul Razak R Kaladgi and Mohammed Samee, Heat transfer augmentation using dimples in forced convection -An experimental approach.Int. J. Mech. Eng. \& Rob. Res. Vol 4, Issue 1, pp 150-153, 2015.

[13] Faheem Akhtar, Abdul Razak R Kaladgi and Mohammed Samee, Heat transfer enhancement using dimple surfaces under natural convection-An experimental study, Int. J. Mech. Eng. \& Rob. Res. Vol 4, Issue 1, pp 173-175, 2015.

[14] Yu Rao ,Yamin Xu,Chaoyi Wan,A Numerical Study of the Flow and Heat Transfer in the Pin Fin-Dimple Channels With Various Dimple Depths, Journal of Heat Transfer, Transactions of the ASME, Vol. 134, pp-071902-1-9, 2012.

[15] P.M. Ligrani, J.L. Harrison, G.I. Mahmood, M.L. Hill, Flow structure due to dimple depression on a channel surface, Phys. Fluids 13 (2001) 3442-3451.

[16] Hasibur Rahman Sardar and Abdul Razak Kaladgi Forced Convection Heat Transfer Analysis through Dimpled Surfaces with Different Arrangements, American Journal of Energy Engineering, Vol 3, Issue 3, pp. 37-45, 2015. 